\title{
La multiculturalidad en la enseñanza de la Historia y el desajuste entre intenciones educativas y prácticas escolares: los retos del presente y del futuro inmediato
}

\section{The multi culture in the History teaching and the umbalance between the educative intentions and school practices: the challenges of the present and immediate future}

A multiculturalidade no ensino da História e o desajuste entre as intenções educativas e as práticas escolares: os desafios do presente e do futuro imediato

\author{
Rafael Valls Montés ${ }^{1}$
}

\begin{abstract}
RESUMEN
En nuestra aportación desarrollamos una aproximación inicial a los conceptos de dimensión europea, dimensión intercultural y de multiculturalidad en relación con la enseñanza de la historia. Nos centramos, seguidamente, en las características que estas dimensiones presentan en la realidad y práctica escolares, mostrando sus principales insuficiencias y, ocasionalmente, deficiencias. Finalmente abordamos los resultados de diversas investigaciones sobre las representaciones y actitudes de los jóvenes escolares españoles, ampliables a los de otros países, respecto de los
\end{abstract}

${ }^{1}$ Profesor titular en el Departamento de Didáctica de las Ciencias Sociales de la Universidad de Valencia (ES). Colaborador en investigaciones del Georg-Eckert-Institute (Braunschweig) e del Proyecto MANES (UNED-Madrid). e-mail: rafael.valls@uv.es 
"extranjeros" y los retos que éstas presentan para una enseñanza orientada a la formación de unos ciudadanos más abiertos, plurales y solidarios.

Palabras clave: multiculturalidad; dimensión europea e intercultural; enseñanza de la Historia, conocimientos escolares; representaciones escolares.

\begin{abstract}
In our contribution we have developed an initial proximity with the concepts of European dimension, intercultural dimension and of multi culture in relation to the history teaching. We have focused on, then, the characteristics which these dimensions have in the school reality and practice, showing its principal insufficiencies and, occasionally, deficiencies. Finally, we have analysed the results of several enquires about the Spanish school youngest' representations and attitudes, expanded to the ones of other countries, respect to the "foreigners" and the challenges which these have for an oriented teaching for the formation of some citizens more opened, plurals and sympathetic.
\end{abstract}

Keywords: multi culture; european and intercultural dimension; History teaching; school knowledge; school representations.

\title{
RESUMO
}

Em nossa proposta desenvolvemos uma aproximação inicial aos conceitos de dimensão européia, dimensão intercultural e de multiculturalidade e sua relação com o ensino de história. Centramos nosso foco, principalmente, nas características que essas dimensões apresentam na realidade e na prática escolares, mostrando suas principais insuficiências e, ocasionalmente, deficiências. Finalmente, abordamos resultados de diversas investigações sobre as representações e atitudes dos jovens escolares espanhóis, que podem ser ampliadas a outros países, a respeito dos "estrangeiros" e os desafíos que estas representações apresentam para um ensino orientado à formação de cidadãos mais abertos, plurais e solidários.

Palavras-chave: multiculturalidade; dimensão européia e intercultural; ensino de História; conhecimentos escolares; representações escolares.

\section{Introducción}

Es una cuestión prácticamente indudable que la enseñanza de la historia y de la geografía en los países occidentales se ha abierto, al menos durante los 
últimos veinte años, a nuevos referentes sociales y culturales internacionales, que sobrepasan los límites del patriotismo en su versión más restringida. En ese sentido se puede afirmar que una dimensión europea, iberoamericana o mediterránea, intercultural, en suma, de estas enseñanzas ya está más presente en los centros escolares occidentales, incluidos los españoles. Y así se comprueba analizando los manuales escolares actuales, especialmente por lo que hace referencia a la dimensión europea y algo menos en relación con la intercultural. Pero, de manera simultánea, también se detecta, tanto en el profesorado como en el alumnado, ignorancias y actitudes no muy favorables a estas nuevas dimensiones de la enseñanza de las ciencias sociales. Por este motivo hemos utilizado el término reto en el título de nuestra aportación, en la que desarrollamos, en primer lugar, una aproximación conceptual a los conceptos de dimensión europea y de dimensión intercultural. Posteriormente nos centramos en las características que ambas presentan en la realidad y práctica escolares, a partir del caso español, mostrando sus principales insuficiencias y, ocasionalmente, deficiencias. Finalmente abordamos los resultados de diversas investigaciones sobre las representaciones y actitudes de los jóvenes escolares españoles respecto de los "extranjeros" y los retos que éstas presentan para una enseñanza orientada a la formación de unos ciudadanos abiertos, plurales y solidarios.

\section{La deseable complementariedad de las dimensiones europea, intercultural y mundial}

Europa está experimentando cambios muy importantes en la actualidad, que no siempre se corresponden con la representación más general o popular que de ella se suele tener. Son cambios que están redefiniendo la realidad europea en prácticamente la totalidad de sus ámbitos.

Al proceso de integración iniciado por los principales países de la Europa Occidental después de la Segunda Guerra Mundial, con sus avances, parones y crisis, se ha unido, por una parte, el cambio producido en el conjunto de los países del Este europeo, tras la desintegración de la Unión Soviética, y el deseo mostrado por la mayoría de estos países de entrar a formar parte, en el futuro más inmediato posible, de la ya constituida Unión Europea. Por la otra, la transformación de una gran parte de las sociedades europeas en receptoras de inmigración, procedente de casi todas las partes del mundo, cuando aquéllas habían sido sociedades con una destacada componente emigratoria durante los últimos cuatro siglos y hasta tiempos bastante recientes.

El proceso de unificación europea es un proyecto de enorme transcendencia, en todos los sentidos, dado que ya afecta, y aún lo hará más en un próximo 
futuro, a la inmensa mayoría de los elementos que definen las condiciones de vida de cualquier tipo de sociedad, en este caso, de las europeas. Es, igualmente, un proceso abierto al futuro, que deberá ser decidido, en gran manera, por la generación de los alumnos que actualmente se están formando. Es de primordial importancia, por tanto, que los alumnos puedan conocer, con la mayor profundidad posible, las características de este proceso abierto y las posibles ventajas e inconvenientes o dificultades que éste comportará y que, ciertamente, influirá de múltiples maneras en su propia vida y en las condiciones en que ésta se tenga que desarrollar.

La creación de una ciudadanía europea, con la consiguiente aceptación de los derechos y deberes a que ésta da lugar, es una de las realidades con la que nuestros alumnos van a contar y en la que van a tener que expresar sus preferencias y, por tanto, decidir democráticamente el tipo de Europa que desean.

La configuración y el impulso de la dimensión europea en la educación supone también un cambio, o al menos una ampliación muy importante, en las tradicionales finalidades de la enseñanza de la historia y de la geografía, o de las ciencias sociales si se quiere, que son las materias en las que centramos nuestro análisis. Estas disciplinas escolares han desempeñado un papel primordial en la configuración de las identidades nacionales, a las que se añadía, sin demasiada convicción práctica, una dimensión mundial que, en función de los intereses nacionales, era valorada de una manera u otra.

El proceso de integración y de impulso de una identidad europea, que en España se complementa con el de las renovadas identidades regionales y de las nacionalidades históricas, plantea unas nuevas y diversificadas formas de identidad, sin necesidad, por el momento, de entrar en la discusión de si las identidades pueden ser sentidas de manera más o menos equivalente, aunque jerarquizada y plural, o si, por el contrario, sólo existe una identidad central y todas las otras son periféricas o secundarias.

En el caso europeo, esta situación de las distintas identidades es especialmente compleja tanto por el enorme empeño desarrollado por las distintas instituciones estatales, fundamentalmente en el siglo XIX y la primera mitad del $\mathrm{XX}$, en la construcción de los imaginarios nacionales respectivos como por la gran variedad de las culturas que han persistido entre las poblaciones europeas.

En este sentido, la configuración de una identidad europea, complementaria de las previamente existentes, tendrá forzosamente que basarse, como mínimo, en una aceptación abierta de la multiculturalidad de tal identidad europea, que las incluya a todas, sin ningún ánimo de homogeneizarlas (RYBA, 1996).

El problema teórico de una posible multiculturalidad es que ésta no comporta necesariamente la aceptación profunda de tal diversidad cultural sino 
que puede convertirse en una marginación encubierta de los culturalmente distintos y, por tanto, en una posible minusvaloración de aquellos que estén incluidos en las culturas que sean consideradas menos representativas o más débiles.

De ahí nuestra opción por el impulso de una dimensión europea que, sin perder su propia especificidad, sea complementaria de una dimensión intercultural abierta al intercambio y mutua influencia del conjunto de las culturas, en primera instancia a aquellas existentes sobre el territorio de la vieja Europa, $y$, en definitiva, a todas aquellas que constituyen el patrimonio conjunto de la humanidad, aunque este planteamiento no tiene porqué ser exclusivamente orientado hacia una interculturalidad global, sino que acepta aproximaciones regionales más parciales como las ya indicadas como las de una dimensión iberoamericana o mediterránea, por poner sólo dos ejemplos ya en marcha, a los que podrían añadirse muchos otros.

¿Pueden las sociedades desarrolladas, especialmente complejas en su articulación, establecer una identidad razonable? La pregunta, formulada por el filósofo Jürgen Habermas (1983) hace ya más de 25 años, sigue siendo una referencia central para las cuestiones que nos ocupan en relación con las dimensiones europea e intercultural en la enseñanza. El epíteto "razonable" es fundamental en cuanto que indica que existen identidades no razonables, que tienden intrínsecamente a delimitar y a excluir. La alternativa no puede ser negar la necesidad humana de establecer identidades individuales y colectivas, sino la de averiguar y desarrollar las formas adecuadas para que éstas puedan enriquecerse mutuamente.

Dado el carácter ambivalente de las posibles identidades, una de las exigencias básicas de la enseñanza de las dimensiones europea e intercultural debe ser la de reflexionar de manera crítica sobre las identidades propias y las ajenas. La necesidad de una actitud autocrítica es especialmente urgente en lo que atañe a la historia, en cuanto que su enseñanza ha formado parte tradicionalmente de la configuración identitaria de las distintas sociedades en la que suelen estar incluidos y entremezclados elementos cognitivos, imaginarios y emocionales (NUSSBAUM, 1999; DE LUCAS, 2003).

Desde el concepto de identidad razonable se pretende romper el circulo vicioso de la pretensión excluyente de la identidad para ubicarla en un contexto de competencia de identidades, en el que quedarían incluidas tanto las múltiples identidades que integra cada individuo en sus diferentes ámbitos de vida, como los cambios de identidades a través del tiempo y de los contextos regionales, nacionales e internacionales de las identidades colectivas, sustrayéndolas de la lógica de la contraidentificación. 
En la búsqueda de posibles soluciones a los tradicionales desarrollos etnocéntricos de la enseñanza de las ciencias sociales, algunos didactas han propuesto convertir el estudio de la historia universal como el punto de partida del conjunto del aprendizaje histórico del alumnado. A través del estudio y análisis de la variedad existida y existente de distintas culturas y sociedades, que es uno de los objetivos básicos de las Ciencias Sociales, los alumnos pueden adquirir formas ampliadas y menos etnocéntricas de identificación, dado que mediante la mirada hacia lo otro y los otros se puede aprender más sobre la propia cultura, con sus posibilidades y sus límites, que con una orientación ensimismada en lo propio (CAJANI, 2008).

Un enfoque de este tipo puede contribuir eficazmente a desarrollar en los alumnos una mayor sensibilidad para el futuro en un momento, como el actual, de aceleración de la historia universal. Este planteamiento pretende, igualmente, poner de manifiesto la interdependencia económico-ecológica universal, así como las cuestiones y problemas en los que toda la humanidad está concernida y que requieren el desarrollo de nuevas formas de responsabilidad y de una ciudadanía o lealtad mundiales (SCHISSLER, 2009).

En nuestra opinión es factible otro tipo de enfoque educativo que haga posible la combinación entre ambas dimensiones, la europea y la intercultural o mundial. El carácter intercultural de la educación que se pretende con el impulso de la dimensión europea de la enseñanza, cuando ésta tiene lugar en situaciones en las que están presentes inmigrantes o minorías de proveniencia extraeuropea, puede y debe estar definido por una dimensión mundial de la misma, de forma que la diversidad cultural presente en cada sociedad pueda ser asumida en todas las variantes que ésta pueda tener en cada momento y, por tanto, con la aceptación de todas sus diferencias.

La dimensión europea en la enseñanza, por tanto, no debe ser entendida, de forma reduccionista y simplificadora, como un intento de crear consenso exclusivamente en torno al proyecto político de la unificación europea. La dimensión europea no debe significar, en este sentido, la creación de un nuevo etnocentrismo, eurocentrismo en expresión más exacta, ni la sustitución de los patriotismos nacionales tradicionales por un nuevo nacionalismo europeísta, sino que comporta igualmente una básica dimensión intercultural, abierta al conjunto de culturas existentes, en la que los ideales de comprensión, respeto y valoración recíprocos, tanto de los aspectos comunes como de las diferencias culturales, sean asumidos de manera clara y decidida.

Proponer una seria reflexión sobre la dimensión europea en la enseñanza de las ciencias sociales presupone una serie de decisiones que no siempre son fáciles de explicitar, especialmente cuando se parte de una actitud crítica de las 
finalidades de la educación en su conjunto. La Unión Europea es una realidad en la que ya vivimos una parte importante de los europeos y, por tanto, su enfoque se puede realizar como una constatación básicamente descriptiva de lo ya existente y de sus características más destacadas.

Se puede plantear, igualmente, desde una perspectiva más dinámica en la que no sólo su pasado tenga un peso destacado, sino en la que su presente y su más o menos inminente futuro entren en consideración, lo que, posiblemente, sea lo más adecuado, pues se correspondería mejor con las necesidades formativoeducativas de los actuales jóvenes escolares, que son los que tendrán que decidir el futuro de tal construcción europea.

También se puede abordar desde una consideración basada en las distintas comprensiones que subyacen a la construcción de la unidad europea y que pueden encaminarse hacia concreciones bastante diversificadas y que, de manera muy esquemática, suelen resumirse en la contrapuesta creación de una Europa de los mercaderes y/o una Europa de los ciudadanos.

La posición que aquí se defiende se basa en una fuerte convicción europea, pero sin ningún ánimo de hacer retórica europeísta de cualquier tipo. Nuestro planteamiento parte, pues, de una clara voluntad de mostrar tanto lo que la Unión Europea ha dado hasta ahora como de dejar constancia de los principales retos con que ésta se enfrenta en muchos y diferentes temas y problemas ya presentes, pero aun no abordados ni resueltos.

En nuestra convicción, la construcción de una Europa unida adquiere su mayor significación cuando se la considera como una contribución importante a la creación de un mundo más unido, más federalizado y, por tanto, de una humanidad más regida por los principios de la justicia social y más activa frente a la violencia y la desigualdad.

El concepto de dimensión europea no es idéntico al de dimensión intercultural, puesto que se relacionan con presupuestos geopolíticos, culturales o étnicos diferentes, pero su intencionalidad más profunda sí que es coincidente, o al menos complementaria, dado que ambas se están refiriendo a la aceptación positiva de la convivencia pacífica entre distintas identidades culturales o nacionales, aunque sea a diferentes escalas. La dimensión europea está referida obviamente a una escala preferentemente intraeuropea, aunque en ella se pueda diferenciar escalas de distinto tipo según nos estemos refiriendo a dimensiones más locales, regionales, nacionales o internacionales. Todas estas escalas forman parte, en función de su mayor o menor diversidad, de la dimensión intercultural, aunque lo más específico de esta última sea su escala mundial, planetaria.

Todo este conjunto de diferentes escalas requieren la atención de la enseñanza, especialmente de la obligatoria, tal como ha sido reconocido en los 
denominados ejes educativos transversales, pues esta institución es la que debe preparar e impulsar, entre otras, una cultura de la paz, en su sentido más amplio y profundo y no simplemente en su consideración contrapuesta a la beligerancia, esto es, una cultura que supere, o al menos aminore, los prejuicios existentes al respecto (AJA et al, 1999).

El problema actual de las sociedades occidentales ya no es el de si se quiere o no vivir en unas sociedades multiculturales, pues éstas ya lo son en realidad, se acepte ésta de mejor o peor gana, sino que el problema reside en la forma en que se quiera vivir conjuntamente. Estamos en el inicio de un nuevo tipo de sociedades, que, con toda seguridad, incrementarán cada vez más su grado de multiculturalidad en el conjunto de las escalas previamente enumeradas.

Esta nueva situación hace que las cuestiones relacionadas con la interculturalidad deban ser ineludiblemente uno de los puntos de atención prioritarios de la reflexión escolar y educativa, especialmente en los centros en donde estas cuestiones ya estén presentes en la propia aula, pero también en aquellos otros lugares en los que estas situaciones aún no sean las más cotidianas de manera directa.

El grado de interculturalidad de las sociedades desarrolladas del presente, entendiendo esta expresión como presencia y simbiosis de diferencias culturales cada vez más amplias sobre un mismo territorio, irá en aumento mientras no se logre unas condiciones aceptables de vida para el conjunto de las sociedades humanas. Mientras esto no sea así, y no parece que esto se vaya a producir en tiempos más o menos próximos, una gran parte de estas sociedades, aquellas que sufran de manera más desfavorable los efectos de la dualización actual del mundo, se verán obligadas a emigrar, a desplazarse en busca de esas condiciones "humanas" de vida, reconocidas en los derechos humanos, cuya primera condición de posibilidad es el derecho a la vida, a no morir de hambre, de miseria o de enfermedades ya superables en otros lugares. Las fronteras nacionales o supraestatales, por más que se refuercen, podrán dificultar estos movimientos, pero no podrán impedirlos.

La inclusión de los inmigrantes dentro de la dimensión europea en la enseñanza y la potenciación de la dimensión intercultural en la misma, a través del estudio tanto de las causas globales que motivaron sus desplazamientos como de las dificultades de todo tipo respecto de su inserción en las sociedades receptoras, pueden permitir, de manera didácticamente muy adecuada, la apertura de las mismas a una educación para el desarrollo, entendida ésta última como un análisis crítico de las desigualdades e injusticias existentes en un mundo económicamente globalizado, pero profundamente dualizado en las condiciones de vida de sus habitantes. 
La configuración de una enseñanza basada en los valores de la justicia, la solidaridad, la aceptación positiva de las diferencias culturales y el impulso de la paz, a través de la superación de las desigualdades en las posibilidades reales de desarrollo humano, son las que deben de estar a la base de las distintas, pero totalmente complementarias, dimensiones de la enseñanza que venimos enumerando. Todas ellas forman parte de los llamados ejes transversales de la educación, de aquellos valores que deben modelar la formación cívica, personal y social de los alumnos como ciudadanos responsables en las diversas dimensiones de su existencia, como ciudadanos de un país determinado, también de Europa en nuestro caso, y del mundo.

En este sentido, la dimensión europea, en su significación más extensa, puede convertirse en una oportunidad muy valiosa para poder desarrollar una personalidad más completa y equilibrada en los alumnos y, sin duda, más adecuada a las características previsibles de los tiempos inmediatamente futuros.

Sólo de esta manera, y desde esta perspectiva mundialista, la dimensión europea merece ser objeto prioritario de atención y puede suscitar la dedicación y el esfuerzo de los docentes en cuanto éstos estén interesados en la construcción de una sociedad más abierta y tolerante para las generaciones futuras. Las declaraciones de la Comisión Europea y del Consejo de Europa (1986 y 1995) estableciendo como patrimonio común europeo el respeto y el cumplimiento de los derechos humanos, de la democracia pluralista, de la justicia social, de la solidaridad y de la preeminencia del derecho así lo dejan entender.

La anterior afirmación, sin embargo, no debe llevarnos a confundir las intenciones con la realidad. En la práctica política real de la Unión Europea aún están muy presentes los límites del pensamiento generado por el conjunto de los Estados-naciones que la integran, en el que sólo son considerados plenamente ciudadanos europeos quienes lo son de alguno de los Estados que forman parte de la misma.

En la actualidad, a pesar de las características cada vez más definidamente globales e interculturales de muchos de los aspectos que la configuran, los Estados-naciones siguen siendo considerados barreras "naturales" y, por tanto, legitimadores de exclusión. Y eso sucede a pesar de que, en un mundo definido por la globalidad y la multiculturalidad, la diferencia entre derechos ciudadanos y derechos humanos no se pueda mantener desde un análisis riguroso de su significación más profunda ni menos desde la consideración de que en materia de derechos algo pueda ser considerado "natural" y no una convención establecida de manera más o menos justificada.

La dimensión europea puede suponer, desde esta perspectiva, un planteamiento problematizador de la realidad existente, un conocimiento y una 
investigación crítica de la misma, tanto para los docentes como para los alumnos. Es la forma de contribuir, desde las propias aportaciones, a la construcción de una Europa, y también de una humanidad en su conjunto, no marcada por los intereses de los "mercaderes" ni de las convenciones más tradicionales y exclusivistas, sino definida prioritariamente por los deseos de los "ciudadanos" y de sus opciones políticas y sociales más solidarias.

\section{La dimensión europea, la intercultural y la mundial en la escuela}

La enseñanza en general, y especialmente la obligatoria, se ha dedicado fundamentalmente, hasta tiempos muy recientes, a la formación de los ciudadanos de la propia nación, concentrando sus esfuerzos en todas aquellas materias y actividades que propiciaban una identidad nacional y patriótica tan fuerte y homogénea como fuese posible. Tarea en la que la contribución de la historia y la geografía se consideraron fundamentales

El actual ideal educativo, por el contrario, ya no queda restringido dentro de los límites y confines del propio Estado, sino que se encamina hacia la configuración de un sujeto, de unas personas capaces de moverse en un contexto más plural y a los que se pretende dotar de la capacidad suficiente para saber valorar realidades complejas y orientarse en un mundo en que una gran cantidad de valores están en competencia entre sí. En esta misma dirección, uno de los puntos de mayor atención por parte de los sistemas educativos de las actuales sociedades desarrolladas es potenciar en los alumnos las actitudes que les permitan valorar y afrontar adecuadamente lo nuevo y diverso respecto de lo tradicional, sin por ello desvirtuar la propia identidad y la propia cultura. En este sentido, el carácter más abierto de la educación pretendida puede ser un buen puente que facilite la comprensión entre los distintos grupos culturales presentes en cada conjunto social, sean éstos de ámbito regional o intra-estatal, sean de ámbito europeo o lo sean de procedencia mundial.

El carácter intercultural de la educación que se pretende con el impulso de la dimensión europea de la enseñanza, cuando ésta tiene lugar en situaciones en las que están presentes inmigrantes o minorías de proveniencia extraeuropea, debe estar definido por una dimensión mundial de la misma, de forma que la realidad cultural presente pueda ser asumida (al menos como conocimiento y como debate) en todas las variantes que ésta pueda tener en cada momento y, por tanto, con la posible y deseable aceptación de todas sus diferencias.

En este sentido, la dimensión europea en la enseñanza debe ser considerada como una primera ampliación, o una superación inicial, de los límites estatales que anteriormente marcaron las pautas básicas de la educación. Esta superación 
de los anteriores límites estatales cuenta actualmente con un respaldo jurídico para los ciudadanos europeo-comunitarios y, en tal sentido, ofrece un horizonte real y tangible, menos utópico y abstracto que el de los planteamientos mundialistas, que deben ser obviamente compartidos en su dimensión ideal, pero que poseen un carácter más evanescente o vago, dado que gozan de una menor protección legal y quedan, desgraciadamente, abiertos a situaciones más arbitrarias.

La dimensión europea en la enseñanza, por tanto, no debe ser entendida, de forma reduccionista y simplificadora, como un intento de crear consenso exclusivamente en torno al proyecto político de la unificación europea. La dimensión europea no debe significar, en este sentido, la creación de un nuevo eurocentrismo, ni la sustitución de los patriotismos nacionales tradicionales por un nuevo nacionalismo europeísta, sino que comporta una básica dimensión intercultural, abierta al conjunto de culturas existentes, en la que los ideales de comprensión, respeto y valoración, tanto de los aspectos comunes como de las diferencias culturales, sean asumidos de manera clara y decidida (BOMBARDELLI, 1997).

Sólo de esta manera, y desde esta perspectiva mundialista, la dimensión europea merece ser objeto prioritario de atención y puede suscitar la dedicación y el esfuerzo de los docentes en cuanto éstos estén interesados en la construcción de una sociedad más abierta y tolerante para las generaciones futuras. Las declaraciones de la Comisión Europea y del Consejo de Europa estableciendo como patrimonio común europeo el respeto y el cumplimiento de los derechos humanos, de la democracia pluralista, de la justicia social, de la solidaridad y de la preeminencia del derecho así lo dejan entender.

La anterior afirmación, sin embargo, no debe llevarnos a confundir las intenciones con la realidad. En la práctica política real de la Unión Europea aún están muy presentes los límites del pensamiento generado por el conjunto de los Estados-naciones que la integran, en el que sólo son considerados plenamente ciudadanos europeos quienes lo son de alguno de los Estados que forman parte de la misma.

En la actualidad, a pesar de las características cada vez más definidamente globales e interculturales de muchos de los aspectos que la configuran, los Estados-naciones siguen siendo considerados barreras "naturales" y, por tanto, legitimadores de exclusión. Y eso sucede a pesar de que, en un mundo definido por la globalidad y la interculturalidad, la diferencia entre derechos ciudadanos y derechos humanos no se pueda mantener desde un análisis riguroso de su significación más profunda ni menos desde la consideración de que en materia de derechos algo pueda ser considerado natural y no una convención establecida 
de manera más o menos justificada. La dimensión europea puede suponer, desde esta perspectiva, un planteamiento problematizador de la realidad existente, un conocimiento y una investigación crítica de la misma, tanto para los docentes como para los alumnos. Es la forma de contribuir, desde las propias aportaciones, a la construcción de una Europa definida prioritariamente por los deseos de los "ciudadanos" y por sus opciones políticas y sociales más solidarias.

En opinión de algunos de los más relevantes expertos sobre la problemática de los inmigrantes en el conjunto de España, la institución escolar ha sido una de las más accesibles para la población extranjera, tanto proveniente de la Unión Europea como extracomunitaria.

El derecho a la educación ha estado garantizado para todos, a pesar de la precariedad jurídica de la mayor parte del colectivo de los extracomunitarios, tal como ha reconocido legalmente el reglamento de 1996 sobre los extranjeros en España. Por los datos disponibles se sabe que, a pesar de su relativamente reducida importancia numérica en el conjunto del sistema educativo, su volumen crece progresivamente, especialmente por lo que respecta a los hijos de los inmigrantes provenientes del Tercer Mundo.

¿Cuál es el tratamiento predominante que se da a tal diversidad cultural en las escuelas españolas?, ¿predominan las tendencias a su asimilación, tolerancia, compensación o a su promoción? Es muy difícil dar respuestas suficientemente documentadas a estas preguntas, ya que esta problemática no ha sido objeto de un debate amplio y continuado. Tal vez la experiencia que ha contado con una mayor continuidad haya sido la realizada, especialmente en la escuela pública, con los jóvenes de la comunidad gitana. Y los resultados no parecen ser optimistas.

Desde una perspectiva teórica se ha distinguido cinco posibles actitudes u opciones del conjunto de la comunidad educativa ante esta cuestión, que van, en sus extremos más opuestos, desde el rechazo de los diferentes culturalmente hasta los impulsores de la denuncia y de la lucha contra el conjunto de los mecanismos generadores de prácticas racistas o discriminatorias (COLECTIVO IOE, 1999).

Una primera actitud es la del rechazo a los diferentes. La diversidad es percibida como un peligro que conviene eludir (dentro de la ancestral concepción de los extranjeros, e incluso de los extraños, como objetos de sospecha, frente a la de los vecinos o conocidos que, por el contrario, gozan habitualmente de presunción de inocencia). Consiguientemente se opta por poner trabas y dificultarles su acceso a la escuela y, si esto no es posible, se intenta reducir al mínimo los contactos con ellos. Esta actitud puede darse tanto entre los directivos o responsables de las escuelas como entre los padres de los alumnos, que 
aconsejan a sus hijos el evitar la proximidad con los diferentes y que, en caso de que su presencia sea considerada excesivamente numerosa, deciden trasladar de centro escolar a los mismos.

La segunda opción es la ignorar a los diferentes. Desde esta representación se considera que el conjunto del alumnado es homogéneo y que existe tanto un modelo único de alumno como de currículo. Puede incluso que, desde perspectivas pretendidamente universalistas, se justifique esta opción desde la no discriminación, en el sentido de que todos los alumnos son iguales y que, por tanto, no deben ser objeto de un trato diferenciador o que atienda a sus peculiaridades culturales específicas. El programa y la práctica escolares, en esta opción, deben seguir las propias pautas establecidas con carácter general, a las que los alumnos deben de adaptarse. Este modelo puede recordar, de manera bastante patente, aunque posiblemente inconsciente, la vertiente autoritaria y homogeneizadora de la escuela tradicional nacionalizadora.

La tercera variante es aquella que ya parte del reconocimiento explícito de la necesidad de educar a los diferentes culturalmente, aunque no por ello modifique los planteamientos generales de la escuela, pero sí abre los espacios y posibilita las condiciones suficientes para que los alumnos diferentes, mediante una atención particularizada, puedan adaptarse al funcionamiento general del conjunto de los alumnos del grupo. Esta opción es la que se plasma habitualmente en la defensa de una educación compensatoria para los alumnos que presentan algún tipo de dificultad, sea ésta de carácter cultural o de cualquier otro. Es también la actitud que actualmente parece contar con más apoyos por parte de los docentes, al menos por lo que se refiere a las enseñanzas infantil y primaria.

La cuarta opción, identificada habitualmente como la de la educación intercultural, es la que hemos venido definiendo a lo largo de los apartados anteriores. En ella se parte de la adaptación del currículo en función del conjunto del alumnado e incorporando elementos provenientes de diferentes perpectivas socioculturales. Este enfoque es relativamente reciente en la escuela española y cuenta aún con pocos materiales didácticos y experiencias docentes, sea por causa de la escasa presencia de diversidades culturales reconocidas por la propia escuela, sea por el peso de una tradición escolar homogeneizadora hasta tiempos bastante recientes.

La quinta variante, identificada habitualmente en distintos ámbitos europeos como educación anti-racista, es muy similar a la educación intercultural, pero le añade una importante componente de tipo económico y sociopolítico a la hora de analizar la exclusión de determinados grupos socioculturales, que, en su consideración, están ausentes de la opción intercultural. Desde la perspectiva anti-racista se considera que la escuela, incluso cuando ésta desarrolla actitudes 
interculturales y genera valores compartidos, no es el lugar donde las posibles exclusiones se puedan obviar o limitar, sino que la marginación y la exclusión tienen su origen en otras instancias más directamente políticas y económicas y que, por tanto, es en estos últimos centros reales de poder en donde hay que actuar, más allá de la conveniencia indudable, pero también de los claros límites, de una educación de características interculturales. Como habrá podido comprobarse, de las cinco variantes reseñadas sólo las dos últimas abordan el tratamiento de la diversidad desde una perspectiva no etnocéntrica, dado que asumen la existencia de un pluralismo cultural e intentan proponer modelos educativos con los que superar las posibles discriminaciones resultantes.

La principal diferencia entre las dos últimas reside en que el planteamiento intercultural acepta, en sus rasgos más generales, el sistema sociopolítico existente y considera que éste puede ser mejorado, entre otras posibilidades, a través de la elaboración de valores culturales compartidos entre los diversos grupos. En este sentido se parte del convencimiento de que tanto el racismo como la xenofobia son fenómenos con una importante componente de índole psicológica y de que están basados en la ignorancia o el desconocimiento y que, por tanto, pueden ser superados, o al menos paliados, mediante una educación adecuada, que esté centrada en la consecución de una valoración igualitaria de las distintas concreciones culturales coexistentes. Por el contrario, la variante anti-racista parte de la consideración de que una educación en valores no es suficiente para hacer frente a los fenómenos racistas o xenófobos, ya que estos responden a las características propias de una organización social basada en la dominación y en la explotación, que persistirán mientras no se consiga una auténtica igualdad de oportunidades para todos los grupos culturales o étnicos.

Estas importantes diferencias analíticas que hemos esbozado no suponen, sin embargo, que ambas variantes no puedan colaborar estrechamente en la configuración de una nueva educación intercultural, que tome como punto de partida la diversidad y la pluralidad realmente existentes en las aulas, para intentar establecer o potenciar el mayor número posible de valores culturales compartidos.

En este sentido, tanto las declaraciones de los responsables educativos de la Unión Europea como del Consejo de Europa y de las distintas instancias educativas del Estado español son convergentes y han manifestado el reconocimiento positivo de tal diversidad cultural y la conveniencia de su tratamiento en las escuelas.

Otra cosa distinta es la repercusión que estas propuestas han tenido en los manuales escolares más usados en los centros educativos (VALLS, 2005) o la actitud mayoritaria del profesorado con respecto a las cuestiones interculturales aquí abordadas (PINGEL et al, 1994 y 2003; PRATS et al, 2001). 
Las posiciones mayoritarias de los docentes españoles parecen estar orientadas hacia las variantes segunda y tercera, esto es, hacia los posicionamientos que intentan evitar los tratamientos desiguales respecto del alumnado, pero sin abordar positivamente el trato de la diversidad, lo que con el tiempo, si no se asume de manera más consciente y efectiva, puede provocar el rechazo por parte de los "diferentes" y su sentimiento de exclusión, a la par que actitudes de violencia de distinto tipo y procedencia como respuesta a tal marginalización o al sentimiento de inseguridad y de peligro frente al diferente.

Estos problemas de interculturalidad ya se están dando en aquellas sociedades más avanzadas en las que la diversidad cultural se ha presentado con mayor intensidad que en España, en donde, con la excepción de la cultura gitana, es una cuestión relativamente reciente y de menor intensidad que en otros países comunitarios, pero que, como dijimos previamente, todos los indicadores demográficos y socioeconómicos muestran que irá en aumento.

En la actualidad, los materiales curriculares existentes sólo suelen abordar, en el mejor de los casos, el tratamiento compensatorio de las diferencias observadas en las aulas, entendidas éstas como deficiencias de algún tipo de conocimientos o de habilidades básicamente lingüísticas, lo que es, evidentemente, un aspecto muy importante de esta cuestión. Pero el problema que aquí planteamos es distinto, pues partimos de que una cultura homogénea, la del grupo dominante, no es la respuesta democráticamente más adecuada a las diferencias existentes en el conjunto de la sociedad.

\section{Los conocimientos de los escolares sobre la geografía e historia de Europa y del mundo}

Son muy escasos los estudios relativos a la relación entre los conocimientos manifestados por los jóvenes y los contenidos estudiados durante su escolarización. Acerca de esta cuestión sólo contamos con estudios generales que valoran los aprendizajes en el conjunto de la Geografía, la Historia y otras ciencias sociales. Las investigaciones específicas, hasta tiempos muy recientes, se centraron casi exclusivamente en los contenidos presentes en los manuales escolares. Algunas fueron promovidas por el Consejo de Europa y tenían como objetivo eliminar de los mismos aquellos estereotipos, expresiones o enfoques que resultasen inaceptables, insultantes o descalificadores respecto de otros países o sociedades. Esta es una práctica que tiene antecedentes importantes ya desde principios del siglo XX, aunque su mayor desarrollo se dio a partir del final de la II Guerra Mundial y ha continuado hasta la actualidad, contando incluso con 
centros especializados en esta labor de análisis comparado de manuales y de propuesta de mejora de los mismos (el principal centro europeo dedicado a este tipo de estudios es el Georg-Eckert-Institut de Braunschweig-Alemania-, que realiza habitualmente los estudios propuestos por el Consejo de Europa y por la UNESCO sobre estas cuestiones. Sus publicaciones sobre los manuales escolares europeos de geografía e historia son muy abundantes y valiosas).

La investigación más importante realizada, dada la amplitud de la muestra utilizada y la inclusión en la misma de la casi totalidad de los países europeos, fue publicada en 1997, aunque sólo una de las cuestiones planteadas a los alumnos hacía referencia directa a su comprensión de la idea de Europa (Angvik-Borries, 1997).

Los principales resultados obtenidos sobre los alumnos españoles ponen de manifiesto que estos jóvenes escolares tienen escasos conocimientos sobre algunos aspectos geográficos básicos como, por ejemplo, los de la localización cartográfica de los países europeos y los de otros países del mundo con sus respectivas capitales. También indican que estos conocimientos son aún menores en lo que respecta a los principales acontecimientos de la historia de Europa y mucho más en lo que respecta a los otros países y continentes del mundo. Asimismo señalan que la presencia de los estereotipos tradicionales sobre los distintos países, europeos y no europeos, siguen vigentes en la mente de estos estudiantes, a pesar de que, como se ha comprobado en diversas investigaciones, tales prejuicios y descalificaciones no tengan ninguna relación con los manuales escolares dada su total ausencia en los mismos.

Un reciente estudio sobre los escolares franceses ha mostrado, por su parte, la escasa relación existente entre los conocimientos adquiridos a través de la enseñanza escolar y el uso que de los mismos hacen los escolares franceses (y lo mismo puede afirmarse en relación con los españoles) a la hora de pronunciarse sobre los problemas o las cuestiones relacionadas con la actual construcción de una Europa unida, con la evolución reciente de la ciudadanía europea y también con respecto a sus vivencias y representaciones de la interculturalidad. Según esta investigación, el estudio de Europa en las aulas no se apoya suficientemente en la experiencia y en las preguntas de los jóvenes, por lo que éstos, a la hora de manifestar sus opiniones, suelen recurrir a sus propias vivencias y al sentido común dominante en la sociedad, pero no a los conocimientos que reciben en las aulas. La conclusión principal de este estudio es que si lo que se pretende con la enseñanza es hacer reflexionar a los alumnos, hacer que se planteen preguntas y que pongan en tela de juicio sus representaciones iniciales y sus estereotipos sociales, entonces es totalmente necesario un nuevo tipo de enseñanza en la que tengan entrada las representaciones de los alumnos, sus 
imaginarios, sus afectos y emociones y no sólo el pretendido carácter neutro de la ciencia, que ellos aceptan como tal, pero que no incorporan a sus vidas ni les ayuda a superar los estereotipos y simplificaciones socialmente más aceptados (TUTIAUX-GUILLON, 2001 y 2009). Algunas recientes investigaciones españolas dan resultados muy semejantes a las otras investigaciones europeas (ESTEPA GIMÉNEZ et al, 2001; PRATS et al, 2000; VALLS et al, 2002).

En general, todos los estudios coinciden en la semejanza entre las visiones de los alumnos y las de la sociedad de sus respectivos países de procedencia, ambas de un marcado carácter convencional respecto de Europa y del resto del mundo. Es muy común, en todos los trabajos reseñados, la alta aceptación que tienen de Europa como lugar de nacimiento de los valores fundamentales de la modernidad (la democracia, la ilustración y el progreso), así como la consideración de la integración europea como el único camino para la paz y para la solución de los problemas económicos y sociales existentes en los distintos países.

\section{Las actitudes de los jóvenes estudiantes españoles respecto de la dimensión europea e intercultural}

Con los datos obtenidos mediante los estudiados previamente reseñados (especialmente PRATS et al. 2001) se puede establecer con suficiente claridad dos niveles de identidad socio-geográficos, políticos y culturales: uno más local e intenso y otro más internacionalizado, de menor intensidad y extensión, y ambos mediados por la identidad española que es la determinante. Desde esta identidad básica, en la que perciben a sus compatriotas españoles como los mejores en muchos aspectos, los jóvenes valoran a los otros europeos y a los ciudadanos de otros países. Para los jóvenes adolescentes, Italia y Francia son los países que más atraen y los italianos son los europeos que se ven con más simpatía y que reciben una mejor valoración. Después de los citados, Gran Bretaña, Alemania, Portugal o los Estados Unidos de Norteamérica serían países apetecibles para ir a vivir, independientemente de que sus ciudadanos no susciten en un porcentaje elevado demasiadas simpatías, especialmente los alemanes y británicos que son vistos con recelo e incluso con una relativa hostilidad. Estos datos confirman lo que se viene señalando de manera reiterada: la Europa que más se conoce, que más se valora y que suscita más sentimientos (en ocasiones encontrados) es la más occidental.

Es decir, los países más próximos, de los que tienen referencias más conocidas, o los que son considerados más parecidos a España, en el que según su visión debe ser más fácil adaptarse y además están suficientemente cerca 
para regresar con frecuencia a sus casas. Estos son los países más elegidos si las circunstancias les hicieran cambiar de residencia. El resto de países europeos y mundiales y sus ciudadanos reciben valoraciones más variadas. Así, por ejemplo, los holandeses son valorados muy bien y los rusos muy mal, aunque también es cierto que a más lejanía el grado de indefinición es mucho más alto.

Como valoración de contraste los jóvenes muestran una gran aceptación e identificación por los estadounidenses a pesar que sus sentimientos hacia ellos son contradictorios. En conjunto los jóvenes españoles les otorgan una valoración más alta que a muchos de sus conciudadanos europeos (alemanes, franceses, portugueses y holandeses). Esta actitud contrasta con la poca aceptación que les suscitan, por ejemplo, los marroquíes.

Para los estudiantes, los estadounidenses son ricos y poderosos y, seguramente, estas características los hacen más atractivos. Otros, los marroquíes, son percibidos como pobres, tienen una lengua, cultura y religión muy distintas y ello, probablemente, los hace menos deseables; incluso se han percibido algunos rasgos que recuerdan posturas hostiles a estos ciudadanos con indicios de una posible e incipiente xenofobia.

Respecto a la aceptación de trabajadores extranjeros en España se puede interpretar que la política restrictiva a la inmigración es una medida que los jóvenes no ven demasiado mal. Es más, piensan que alguna restricción debe darse. De los datos manejados en estas investigaciones parecen desprenderse cuatro conclusiones generales. La primera es que la mayor preocupación de los jóvenes españoles está en su futuro; por ello estarían más de acuerdo en permitir la llegada de inmigrantes si perciben que su entrada no les va restar posibilidades laborales y sociales; la segunda es que están menos de acuerdo que los adultos en atribuir a los inmigrantes comportamientos sociales negativos (delincuencia, marginación o peligrosidad).

En tercer lugar debe señalarse que aquellos jóvenes que ven su futuro de forma menos segura, bien por su posición social o por los problemas que han visto en sus familias, apoyan las medidas más restrictivas respecto de la inmigración.

Y, finalmente, es destacable que ven con normalidad la llegada de europeos de la Unión a ocupar puestos de trabajo en España y que tienen alguna mayor reticencia respecto de los otros extranjeros no comunitarios, aceptando mayoritariamente que ocupen un puesto de trabajo si éstos están más preparados para realizarlo. En esta misma línea, los jóvenes españoles no tienen recelos destacables hacia los europeos de la Unión en el terreno de los derechos civiles, políticos o de otro tipo, sintiéndose más próximos a éstos que al resto de los extranjeros, sean éstos europeos o no. 
La dimensión europea y mundial en los actuales manuales de la E.S.O. (en porcenta

\begin{tabular}{llllll}
\hline editorial & $\begin{array}{l}\text { Europa } \\
\text { general }\end{array}$ & $\begin{array}{l}\text { países } \\
\text { europeos } \\
\text { concretos }\end{array}$ & $\begin{array}{l}\text { Este y } \\
\text { Sureste } \\
\text { europeos }\end{array}$ & España & $\begin{array}{l}\text { Resto } \\
\text { del } \\
\text { mundo }\end{array}$ \\
\hline Santillana & $35^{\prime} 2$ & $19^{\prime} 5$ & $5^{\prime} 2$ & $27^{\prime} 8$ & $11^{\prime} 3$ \\
ECIR & $41^{\prime} 7$ & $10^{\prime} 8$ & $8^{\prime} 3$ & $22^{\prime} 5$ & $16^{\prime} 7$ \\
S.M. & $41^{\prime} 3$ & $10^{\prime} 6$ & $5^{\prime} 6$ & $32^{\prime} 5$ & 10 \\
Vicens-Vives & $44^{\prime} 4$ & $14^{\prime} 8$ & $5^{\prime} 6$ & $18^{\prime} 6$ & $16^{\prime} 6$ \\
Anaya & $38^{\prime} 8$ & $7^{\prime} 2$ & $3^{\prime} 8$ & 38 & $11^{\prime} 2$ \\
\hline
\end{tabular}

Anexo - La distribución cuantitativa de los distintos referentes socio-culturales en los principales manuales españoles de Ciencias Sociales, geografia e historia de la Educación Secundaria Obligatoria (ESO).

Los datos más interesantes de esta tabla son los referidos a Europa, por una parte, y al resto del mundo por la otra. En lo que respecta a Europa, su presencia genérica ha crecido, pero de una manera muy descompensada en lo referido al tratamiento de las dos Europas tradicionales: los manules son muy "occidentalistas", pues tanto la primera como la segunda columna están referidas casi exclusivamente a la Europa Occidental, la Europa más poderosa, mientras que la tercera columna, con valores muy inferiores, es la que se refiere al resto de Europa. Si incluimos a España (en este caso sin diferenciar el porcentaje dedicado a las distintas Autonomías regionales, que supone un porcentaje medio entre el 7\% y el 10\%, según las Autonomías) dentro del conjunto de la Europa Occidental, el resultado es muy significativo, pues supera las tres cuartas partes del total. Pero aún más llamativa, y muy problematizadora, es la escasa presencia de la componente mundial de los manuales (en relación especialmente con los aspectos históricos, que son algo inferiores a los más estrictamente geográficos).

El desequilibrio existente entre estos distintos porcentajes es, obviamente, muy importante: al conjunto del continente americano se le dedica en estos manuales de historia un muy escaso 4,4 por ciento, que se divide en dos partes prácticamente idénticas entre America del Norte y América Latina. Un porcentaje muy similar es el que recibe el conjunto de los países asiáticos y aún menor es la presencia de las sociedades y culturas africanas, presentes tan sólo en un 3 por ciento de los contenidos de estos manuales. La presencia de Oceanía es mínima: el $0^{\prime} 1$ por ciento. 
Estos datos nos hacen pensar que la enseñanza de las ciencias sociales (al menos en lo que los manuales escolares la definen) no ha logrado despegarse suficientemente de sus características etnocéntricas tradicionales, que, en nuestro caso, podrían definirse como occidentalocéntricas y que tanto una dimensión realmente europea como una dimensión mundial y plenamente intercultural, a pesar de los avances realizados en los últimos años, aún son tareas pendientes, además de ser urgentemente necesarias.

\section{REFERENCIAS}

AJA, E. ET AL: La inmigració estrangera a Espanya: els reptes educatius. Barcelona, Fundació La Caixa, 1999.

ANGVIK, MAGNE y BODO VON BORRIES: A comparative european survey on historical consciousness and political attitudes among adolescents. Hamburg, Körber Stiftung, 2 vols, 1997.

BOMBARDELLI, OLGA: Formazione in dimensione europea e interculturale. Brescia, Editrice la Scuola, 1997;

CAJANI, LUIGI: "La mirada sobre el otro: el Islam y Europa en sus manuales de historia" en Luigi Cajani (director): Conociendo al otro: el Islam y Europa en sus manuales de historia. Madrid, Santillana, 2008, pp. 7-36.

COLECTIVO IOE: Inmigrantes, trabajadores, ciudadanos. Una visión de las migraciones desde España. Valencia, Universitat de València-Patronat SudNord., 1999.

CONSEJO DE EUROPA: Contre les stéréotipes et les préjugés. Les travaux du Conseil de l'Europe sur l'enseignement de l'histoire et les manuels d'histoire (1953-1994). Strasbourg, Consejo de cooperación cultural, 1986.

: The european content of the school history curriculum.

Strasbourg, Consejo de Europa, 1995.

DE LUCAS, JAVIER: Globalización e identidades. Claves políticas y jurídicas. Barcelona, Icaria, 2003.

ESTEPA GIMÉNEZ, J; FRIERA SUÁREZ, F. Y PIÑEIRO PELETEIRO, R 
(eds): Identidades y territorios: un reto para la didáctica de las Ciencias Sociales. Oviedo, Krk Ediciones, 2001.

HABERMAS, J.: Reconstrucción del materialismo histórico. Madrid, Taurus, 1983.

NUSSBAUM, M. (ed.): Los límites del patriotismo. Identidad, pertenencia y ciudadanía mundial. Barcelona, Paidós, 1999.

PINGEL, FALK ET AL: L'Immagine dell'Europa nei manuali scolastici di Germania, Francia, Spagna, Gran Bretagna e Italia. Torino, Fundación Agnelli, 1994.

PINGEL, FALK ET AL: Insegnare l'Europa. Concetti e rappresentazioni nei libri di testo europei. Torino, Fundación Agnelli, 2003.

PRATS, JOAQUIN (DIR.); TREPAT, C. (COORD.); PEÑA, J. V.; VALLS, R.; URGELL, F. Los jóvenes ante el reto europeo. Conocimientos y expectativas del alumnado de educación secundaria. Barcelona, Fundación La Caixa, 2001.

RYBA, R.: "Le programme de ressources pédagogiques pour la dimension europénne: conception, mise en oeuvre et resultats" en VV.AA: La dimension européenne: rapport de synthèse. Strasbourg, Conseil de 1'Europe, 1996, pp. 36-64.

SCHISSLER, HANNA: "Navigating a globalizing World: thoughts on texbook análisis, teaching and learning" en Contexts. The journal of educational Media, Memory and Society, 1, 2009, pp.203-226.

TUTIAUX-GUILLON, NICOLE: L'Europe entre project politique et objet scolaire. Paris, Institut National de la Recherche Pédagogique, 2001

TUTIAUX-GUILLON, NICOLE: "La citoyennité européenne, une inconnue de la formation en France?" en Rosa María Ávila, B. Borghi e I. Matozzi: La educación de la ciudadanía europea y la formación del profesorado. Bologna, Pàtron editore, 2009, pp.447-457.

VALLS, R. Y LÓPEZ, A. (eds.); BROTAT, J; DEL MORAL, C.; PÉREZ, C.; PINGEL, F.; RUEDA, F. Dimensión europea e intercultural en la enseñanza de las ciencias sociales. Madrid, Síntesis, 2002. 
VALLS, RAFAEL: "L'insegnamento della storia contemporanea nella scuola secondaria spagnola: il dibattito attuale e i problemi aperti" en Alessandro Cavalli: Insegnare la storia contemporanea in Europa. Bologna, Il Mulino, 2005, pp. 273-325.

Texto recebido em 03 de novembro de 2010.

Texto aprovado em 17 de maio de 2011. 